\title{
Numerical solution of a Fredholm integro-differential equation modelling neural networks
}

\author{
Z. Jackiewicz*, M. Rahman, B.D. Welfert \\ Department of Mathematics, Arizona State University, Tempe, AZ 85287, USA
}

Available online 25 May 2005

\begin{abstract}
We compare piecewise linear and polynomial collocation approaches for the numerical solution of a Fredholm integro-differential equations modelling neural networks. Both approaches combine the use of Gaussian quadrature rules on an infinite interval of integration with interpolation to a uniformly distributed grid on a bounded interval. These methods are illustrated by numerical experiments on neural networks equations.
\end{abstract}

๑ 2005 IMACS. Published by Elsevier B.V. All rights reserved.

Keywords: Fredholm integro-differential equation; Euler-Hermite and Euler-Laguerre method; Neural networks

\section{Introduction}

We consider the initial-value problem for the integro-differential equation of convolution type

$$
\left\{\begin{array}{l}
\partial_{t} \theta(x, t)=f(\theta(x, t), t)+\mu \int_{-\infty}^{\infty} K(x-y) \partial_{t} \theta(y, t) \mathrm{d} y, \\
\theta(x, 0)=g(x),
\end{array}\right.
$$

$-\infty \leqslant x \leqslant \infty, t \geqslant 0$, with a given smooth function $f$ and an initial function $g$. The kernel $K(s)$ is assumed to be a nonnegative integrable function defined on $\mathbb{R}$. We shall mainly be interested in the choices

$$
K(s)=\frac{1}{\sigma \sqrt{\pi}} e^{-(s / \sigma)^{2}} \quad \text { for } s \in \mathbb{R},
$$

\footnotetext{
* Corresponding author.

E-mail addresses: jackiewi@math.la.asu.edu (Z. Jackiewicz), rahman@mathpost.asu.edu (M. Rahman), welfert@asu.edu
} (B.D. Welfert).

0168-9274/\$30.00 @ 2005 IMACS. Published by Elsevier B.V. All rights reserved. doi:10.1016/j.apnum.2005.04.020 
and

$$
K(s)= \begin{cases}\frac{1}{\sigma} e^{-s / \sigma}, & \text { for } s \geqslant 0, \\ 0, & \text { for } s<0,\end{cases}
$$

for some $\sigma>0$. The problem (1.1) is a continuous analog of a discrete voltage controlled oscillator neuron (VCON) model of transmission line in neural networks discussed in [4,5]. The angle $\theta(x, t)$ represents the phase at time $t$ associated with a neuron located at the point $x$, and the integral appearing in (1.1) corresponds to the influence of neighboring neurons. Kernels of the form (1.2) are associated to bi-directional influence while those of the form (1.3) correspond to unidirectional connectivity. In both cases the influence is stronger between neurons that are close to each other.

Eq. (1.1) describes a one-dimensional chain of single neurons interacting with each other according to the kernel (probability density function) $K$ defined by (1.2) or (1.3). A different approach, based on models describing single neural cells, is presented in $[2,6]$.

A forward Euler time discretization of (1.1) with a stepsize $\Delta t$ yields

$$
\theta_{i+1}(x)=F_{i}(x)+\mu \int_{-\infty}^{\infty} K(x-y) \theta_{i+1}(y) \mathrm{d} y
$$

with

$$
F_{i}(x)=\theta_{i}(x)+\Delta t f\left(\theta_{i}(x), i \Delta t\right)-\mu \int_{-\infty}^{\infty} K(x-y) \theta_{i}(y) \mathrm{d} y
$$

and $\theta_{i}(x)=\theta(x, i \Delta t)$. Eq. (1.4) is a standard Fredholm integral equation of the second kind, for which efficient solution procedures exist, in particular for separable kernel of the form

$$
K(x, y)=\sum_{i=1}^{m} a_{i}(x) b_{i}(y) .
$$

Observe that the kernel (1.3) is of this type with $m=1$. In other cases, such as (1.2), the integral in (1.4) must be approximated using some quadrature rule.

A solution strategy based on the use of Gaussian quadrature rules and interpolation to a regular grid on a bounded interval is described in Section 2. It is in particular shown that special care must be exercised in how interpolation is applied. Note that higher-order time integration schemes also lead to problems of the form (1.4) but with a function $F(x)$ of a different form.

The approach used in Section 3 is somewhat different. We first approximate the function $\theta(x, t)$ using an expansion in terms of Lagrange fundamental polynomials with respect to some abscissas. Then we derive the system of differential equations for the coefficients of this expansion by collocating at some points $x_{j}$ on a bounded interval $[-A, A]$. In both approaches a linear system of equations is solved at each time integration step.

The approach proposed in this paper is also applicable to the numerical solution of models for the spread of bacteriophage infection considered by Gourley and Kuang [3]. 


\section{An Euler-Hermite and an Euler-Laguerre approach}

In this section we will describe the numerical method for (1.1) where the integral term is approximated by the Gauss-Hermite quadrature rule if the kernel $K$ is given by (1.2) or by the Gauss-Laguerre quadrature rule if $K$ is given by (1.3). The problem (1.1) with $K$ given by (1.2) after the change of variables $\eta=(x-y) / \sigma$ takes the form

$$
\left\{\begin{array}{l}
\partial_{t} \theta(x, t)=f(\theta(x, t), t)+\bar{\mu} \int_{-\infty}^{\infty} e^{-\eta^{2}} \partial_{t} \theta(x-\sigma \eta, t) \mathrm{d} \eta, \\
\theta(x, 0)=g(x),
\end{array}\right.
$$

$-\infty<x<\infty, t \geqslant 0$, where $\bar{\mu}=\mu / \sqrt{\pi}$. Similarly, (1.1) with $K$ given by (1.3) after the same change of variables takes the form

$$
\left\{\begin{array}{l}
\partial_{t} \theta(x, t)=f(\theta(x, t), t)+\mu \int_{0}^{\infty} e^{-\eta} \partial_{t} \theta(x-\sigma \eta, t) \mathrm{d} \eta \\
\theta(x, 0)=g(x)
\end{array}\right.
$$

$-\infty<x<\infty, t \geqslant 0$. We will discretize the integral term in (2.1) by the Gauss-Hermite quadrature rule

$$
\int_{-\infty}^{\infty} e^{-x^{2}} f(x) \mathrm{d} x=\sum_{k=1}^{n} w_{k}^{H} f\left(x_{k}^{H}\right)+\frac{n ! \sqrt{\pi}}{2^{n}(2 n) !} f^{(2 n)}(\xi),
$$

$-\infty<\xi<\infty$, and the integral term in (2.2) by the Gauss-Laguerre quadrature rule

$$
\int_{0}^{\infty} e^{-x} f(x) \mathrm{d} x=\sum_{k=1}^{n} w_{k}^{L} f\left(x_{k}^{L}\right)+\frac{(n !)^{2}}{(2 n) !} f^{(2 n)}(\xi),
$$

$0<\xi<\infty$. The rule (2.3) is convergent if the function $f$ satisfies the inequality

$$
|f(x)| \leqslant \frac{e^{x^{2}}}{|x|^{1+\rho}}
$$

and (2.4) is convergent if

$$
|f(x)| \leqslant \frac{e^{x}}{|x|^{1+\rho}}
$$

for some $\rho>0$, compare [1]. Moreover, if $f$ has bounded derivatives of any order then the errors of (2.3) and (2.4) tend to zero as $n \rightarrow \infty$ with spectral rate. This is illustrated in Fig. 1 where we have plotted in double logarithmic scale the ratios $n ! \sqrt{\pi} /\left(2^{n}(2 n) !\right)$ and $(n !)^{2} /((2 n) !)$ versus $n$ for $1 \leqslant n \leqslant 32$.

To approximate (2.1) or (2.2) we restrict the range of the variable $x$ to the symmetric bounded interval $[-A, A]$, where $A$ is large enough, and introduce the grid $x_{j}=-A+(j-1) \Delta x, \Delta x=A / N, j=$ $1,2, \ldots, 2 N+1$. We introduce also the grid $t_{i}=(i-1) \Delta t, \Delta t=T / M, i=1,2, \ldots, M+1$, on the time interval $[0, T]$. Then (2.1) and (2.2) for $x=x_{j}$ and $t=t_{i}$ and with the integral terms discretized by (2.3) and (2.4), respectively, take the form

$$
\begin{aligned}
& \left\{\begin{array}{l}
\partial_{t} \theta\left(x_{j}, t_{i}\right)=f\left(\theta\left(x_{j}, t_{i}\right), t_{i}\right)+\bar{\mu} \sum_{k=1}^{n} w_{k}^{H} \partial_{t} \theta\left(x_{j}-\sigma \eta_{k}^{H}, t_{i}\right), \\
\theta\left(x_{j}, t_{1}\right)=g\left(x_{j}\right),
\end{array}\right. \\
& j=1,2, \ldots, 2 N+1, i=1,2, \ldots, M \text {, and } \\
& \left\{\begin{array}{l}
\partial_{t} \theta\left(x_{j}, t_{i}\right)=f\left(\theta\left(x_{j}, t_{i}\right), t_{i}\right)+\mu \sum_{k=1}^{n} w_{k}^{L} \partial_{t} \theta\left(x_{j}-\sigma \eta_{k}^{L}, t_{i}\right), \\
\theta\left(x_{j}, t_{1}\right)=g\left(x_{j}\right),
\end{array}\right.
\end{aligned}
$$



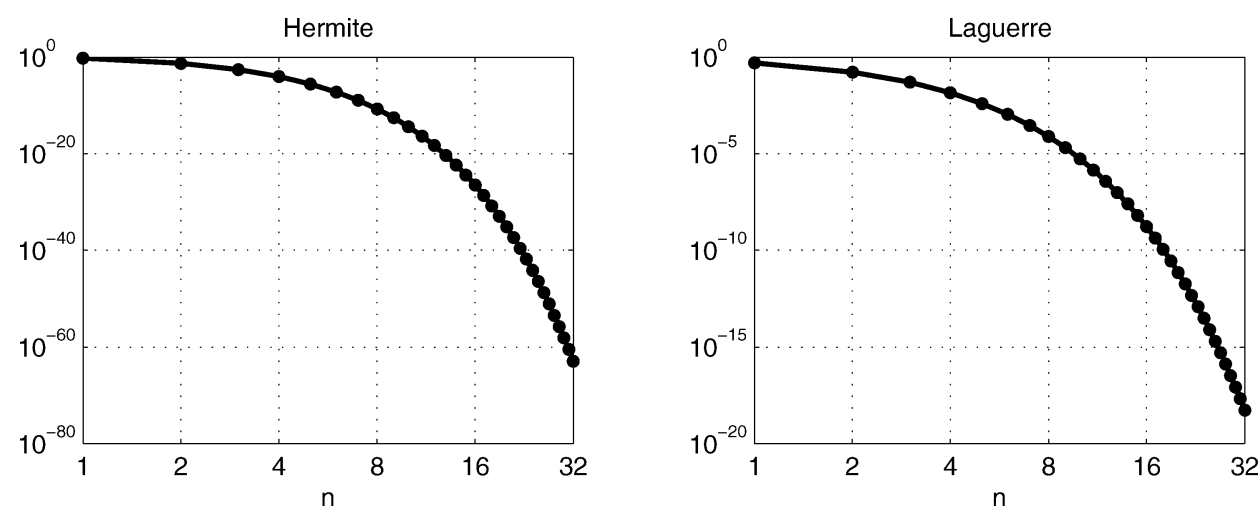

Fig. 1. Ratios $n ! \sqrt{\pi} /\left(2^{n}(2 n) !\right)$ and $(n !)^{2} /((2 n) !)$ versus $n$ for $1 \leqslant n \leqslant 32$.

$j=1,2, \ldots, 2 N+1, i=1,2, \ldots, M$. Here, $w_{k}^{H}, w_{k}^{L}$, and $\eta_{k}^{H}, \eta_{k}^{L}$, are the weights and abscissas of the Gauss-Hermite (2.3) and Gauss-Laguerre (2.4) quadrature rules, respectively, which can be computed using eigenvalue theory of tridiagonal matrices, compare [1].

Observe that the discretizations of (2.1) and (2.2) require the values of $\theta\left(x_{j}-\sigma \eta_{k}^{H}, t_{i}\right)$ and $\theta\left(x_{j}-\right.$ $\left.\sigma \eta_{k}^{L}, t_{i}\right)$ in some layer outside of the interval $[-A, A]$. Observe also that the size of this layer increases with the number of points $n$ in (2.3) and (2.4). The large size of this layer is not desirable so there is a trade-off between the accuracy of the quadrature rules (2.3) and (2.4) and the accuracy of the overall numerical schemes (2.5) and (2.6).

Discretizing $\partial_{t} \theta\left(x_{j}, t_{i}\right)$ and $\partial_{t} \theta\left(x_{j}-\sigma \eta_{k}, t_{i}\right), \eta_{k}=\eta_{k}^{H}$ or $\eta_{k}=\eta_{k}^{L}$, appearing in (2.5) and (2.6) by the Euler method we obtain

$$
\begin{aligned}
&\left\{\begin{array}{l}
\theta_{i+1}\left(x_{j}\right)-\bar{\mu} \sum_{k=1}^{n} w_{k}^{H} \theta_{i+1}\left(x_{j}-\sigma \eta_{k}^{H}\right) \\
\quad=\theta_{i}\left(x_{j}\right)+\Delta t f\left(\theta_{i}\left(x_{j}\right), t_{i}\right)-\bar{\mu} \sum_{k=1}^{n} w_{k}^{H} \theta_{i}\left(x_{j}-\sigma \eta_{k}^{H}\right), \\
\theta_{1}\left(x_{j}\right)=g\left(x_{j}\right),
\end{array}\right. \\
& j=1,2, \ldots, 2 N+1, i=1,2, \ldots, M, \text { and }
\end{aligned}
$$

$j=1,2, \ldots, 2 N+1, i=1,2, \ldots, M$, where $\theta_{i}\left(x_{j}\right)=\theta\left(x_{j}, t_{i}\right)$. We still have to approximate the expressions $\theta_{i}\left(x_{j}-\sigma \eta_{k}\right)$, and $\theta_{i+1}\left(x_{j}-\sigma \eta_{k}\right)$, using the values of $\theta_{i}$ and $\theta_{i+1}$ defined only at the grid points $x_{j}$. If $x_{j}-\sigma \eta_{k} \leqslant x_{1}=-A$, then for any $t$ we extrapolate $\theta\left(x_{j}-\sigma \eta_{k}, t\right)$ using the formula

$$
\theta\left(x_{j}-\sigma \eta_{k}, t\right)=\left(1-\frac{x_{j}-\sigma \eta_{k}-x_{1}}{\Delta x}\right) \theta\left(x_{1}, t\right)+\frac{x_{j}-\sigma \eta_{k}-x_{1}}{\Delta x} \theta\left(x_{2}, t\right) .
$$

If $x_{1}<x_{j}-\sigma \eta_{k}<x_{2 N+1}$ then we use piecewise linear interpolation

$$
\theta\left(x_{j}-\sigma \eta_{k}, t\right)=\left(1-\frac{x_{j}-\sigma \eta_{k}-x_{q-1}}{\Delta x}\right) \theta\left(x_{q-1}, t\right)+\frac{x_{j}-\sigma \eta_{k}-x_{q-1}}{\Delta x} \theta\left(x_{q}, t\right),
$$


where $x_{j}-\sigma \eta_{k} \in\left(x_{q-1}, x_{q}\right]$. This corresponds to the index $q$ defined by

$$
q=\operatorname{ceil}\left(\frac{x_{j}-\sigma \eta_{k}-x_{1}}{\Delta x}\right)+1 .
$$

If $x_{j}-\sigma \eta_{k} \geqslant x_{2 N+1}=A$ (this is applicable only if $\eta_{k}=\eta_{k}^{H}$ since $\eta_{k}^{L}>0$ ) then we extrapolate $\theta\left(x_{j}-\right.$ $\left.\sigma \eta_{k}, t\right)$ according to the formula

$$
\theta\left(x_{j}-\sigma \eta_{k}, t\right)=\left(1-\frac{x_{j}-\sigma \eta_{k}-x_{2 N+1}}{\Delta x}\right) \theta\left(x_{2 N+1}, t\right)+\frac{x_{j}-\sigma \eta_{k}-x_{2 N+1}}{\Delta x} \theta\left(x_{2 N}, t\right) .
$$

Putting

$$
\bar{\theta}_{i}=\left[\begin{array}{llll}
\theta_{i}\left(x_{1}\right) & \theta_{i}\left(x_{2}\right) & \ldots & \theta_{i}\left(x_{2 N+1}\right)
\end{array}\right]^{\mathrm{T}}
$$

this leads to the linear systems of equations

$$
Q_{H} \bar{\theta}_{i+1}=b_{H}\left(\bar{\theta}_{i}\right),
$$

and

$$
Q_{L} \bar{\theta}_{i+1}=b_{L}\left(\bar{\theta}_{i}\right)
$$

at each level of time integration $i=1,2, \ldots, M$, with given initial condition

$$
\bar{\theta}_{1}=\left[\begin{array}{llll}
g\left(x_{1}\right) & g\left(x_{2}\right) & \ldots & g\left(x_{2 N+1}\right)
\end{array}\right]^{\mathrm{T}} .
$$

The matrices $Q_{H}, Q_{L} \in \mathbb{R}^{(2 N+1) \times(2 N+1)}$, and the right-hand sides $b_{H}\left(\bar{\theta}_{i}\right), b_{L}\left(\bar{\theta}_{i}\right) \in \mathbb{R}^{2 N+1}$ appearing in (2.9) and (2.10) can be assembled according to the interpolation procedure described above. The resulting matrices $Q_{H}$ and $Q_{L}$ are sparse and well-conditioned and the linear systems (2.9) and (2.10) can be efficiently and accurately solved taking advantage of sparse matrix technology.

We have also experimented with piecewise constant interpolation. In this procedure $\theta\left(x_{j}-\sigma \eta_{k}^{H}, t\right)$ in (2.7) was approximated by $\theta\left(x_{1}, t\right)$ if $x_{j}-\sigma \eta_{k}^{H} \leqslant x_{1}$, by $\theta\left(x_{q}, t\right)$ if $x_{1}<x_{j}-\sigma \eta_{k}^{H} \leqslant 0$ and $x_{j}-\sigma \eta_{k}^{H} \in$ $\left(x_{q-1}, x_{q}\right]$ or $0 \leqslant x_{j}-\sigma \eta_{k}^{H} \leqslant x_{2 N+1}$ and $x_{j}-\sigma \eta_{k}^{H} \in\left(x_{q}, x_{q+1}\right]$ and by $\theta\left(x_{2 N+1}, t\right)$ if $x_{j}-\sigma \eta_{k}^{H}>x_{2 N+1}$. This means that we interpolate to the right if $x_{j}-\sigma \eta_{k}^{H} \leqslant 0$ and to the left if $x_{j}-\sigma \eta_{k}^{H}>0$. As a result, this procedure introduces discontinuity at the point $x=0$ which has a negative effect on the accuracy of the resulting numerical scheme (2.7). This will be illustrated in Section 4. In the Laguerre case, $\theta\left(x_{j}-\right.$ $\left.\sigma \eta_{k}^{L}, t\right)$ in (2.8) was approximated by $\theta\left(x_{1}, t\right)$ if $x_{j}-\sigma \eta_{k}^{L} \leqslant x_{1}$ and by $\theta\left(x_{q}, t\right)$ if $x_{1}<x_{j}-\sigma \eta_{k}^{L} \leqslant x_{2 N+1}$ and $x_{j}-\sigma \eta_{k}^{L} \in\left(x_{q-1}, x_{q}\right]$. This means that we always interpolate to the right which leads to an acceptable accuracy of the resulting scheme (2.8).

\section{Spectral collocation approach}

In this section we will look for an approximation to $\theta(s, t)$ in the form

$$
\theta(s, t)=\sum_{\nu=0}^{n} a_{v}(t) \ell_{v}(s / \sigma),
$$

where $\ell_{v}(z)$ are Lagrange fundamental polynomials

$$
\ell_{v}(z)=\prod_{j=0, j \neq v}^{n} \frac{z-z_{j}}{z_{v}-z_{j}}
$$


$v=0,1, \ldots, n$, with respect to the given abscissas $z_{j}$. Putting $x=s / \sigma$ and substituting (3.1) into (1.1) we obtain

$$
\sum_{\nu=0}^{n} a_{\nu}^{\prime}(t) \ell_{\nu}(x)=f\left(\sum_{\nu=0}^{n} a_{\nu}(t) \ell_{\nu}(x), t\right)+\bar{\mu} \sum_{\nu=0}^{n} a_{\nu}^{\prime}(t) \int_{-\infty}^{\infty} e^{-(x-y)^{2}} \ell_{\nu}(y) \mathrm{d} y,
$$

$\bar{\mu}=\mu / \sqrt{\pi}$, if the kernel $K$ is given by $(1.2)$, or

$$
\sum_{\nu=0}^{n} a_{\nu}^{\prime}(t) \ell_{\nu}(x)=f\left(\sum_{\nu=0}^{n} a_{\nu}(t) \ell_{\nu}(x), t\right)+\mu \sum_{\nu=0}^{n} a_{\nu}^{\prime}(t) \int_{-\infty}^{x} e^{-(x-y)} \ell_{\nu}(y) \mathrm{d} y,
$$

if the kernel $K$ is given by (1.3). Making the change of variables $\eta=x-y$, then approximating the integrals by Hermite or Laguerre quadrature rules (2.3) or (2.4) and putting $x=x_{j}$, where the abscissas $x_{j}$ are defined in Section 2 we obtain

$$
\sum_{\nu=0}^{n}\left(\ell_{\nu}\left(x_{j}\right)-\bar{\mu} \sum_{k=1}^{r} w_{r}^{H} \ell_{\nu}\left(x_{j}-\eta_{r}^{H}\right)\right) a_{\nu}^{\prime}(t)=f\left(\sum_{\nu=0}^{n} a_{\nu}(t) \ell_{\nu}\left(x_{j}\right), t\right),
$$

or

$$
\sum_{\nu=0}^{n}\left(\ell_{v}\left(x_{j}\right)-\mu \sum_{k=1}^{r} w_{r}^{L} \ell_{\nu}\left(x_{j}-\eta_{r}^{L}\right)\right) a_{\nu}^{\prime}(t)=f\left(\sum_{\nu=0}^{n} a_{\nu}(t) \ell_{\nu}\left(x_{j}\right), t\right),
$$

$j=1,2, \ldots, 2 N+1$. Letting

$$
\begin{aligned}
& a(t)=\left[\begin{array}{llll}
a_{0}(t) & a_{1}(t) & \ldots & a_{n}(t)
\end{array}\right]^{\mathrm{T}}, \quad H=\left[h_{j v}\right]_{j=1,2, \ldots, 2 N+1, v=0,1, \ldots, n}, \\
& Q_{H}=\left[q_{j v}^{H}\right]_{j=1,2, \ldots, 2 N+1, \nu=0,1, \ldots, n}, \quad Q_{L}=\left[q_{j v}^{L}\right]_{j=1,2, \ldots, 2 N+1, \nu=0,1, \ldots, n},
\end{aligned}
$$

where $h_{j v}=\ell_{v}\left(x_{j}\right)$,

$$
q_{j v}^{H}=h_{j v}-\bar{\mu} \sum_{k=1}^{r} w_{r}^{H} \ell_{\nu}\left(x_{j}-\eta_{r}^{H}\right), \quad q_{j v}^{L}=h_{j v}-\mu \sum_{k=1}^{r} w_{r}^{L} \ell_{v}\left(x_{j}-\eta_{r}^{L}\right),
$$

(3.2) and (3.3) can be written in the following vector form

$$
Q a^{\prime}(t)=f(H a(t), t),
$$

$t \geqslant 0, Q=Q_{H}$ or $Q=Q_{L}$. The initial condition $a_{1}=a\left(t_{1}\right)=a(0)$ can be obtained from the equation

$$
H a_{1}=G,
$$

where

$$
G=\left[\begin{array}{llll}
g\left(\sigma x_{1}\right) & g\left(\sigma x_{2}\right) & \ldots & g\left(\sigma x_{2 N+1}\right)
\end{array}\right]^{\mathrm{T}} .
$$

These systems are then solved by the forward Euler method

$$
Q a_{i+1}=Q a_{i}+\Delta t f\left(H a_{i}, t_{i}\right),
$$

$i=1,2, \ldots, M$, where (3.6) is solved on each time level by Gaussian elimination if $n=2 N$ or least squares minimization if $n>2 N$. Here, $M$ and $\Delta t$ are the same as in Section 2. 

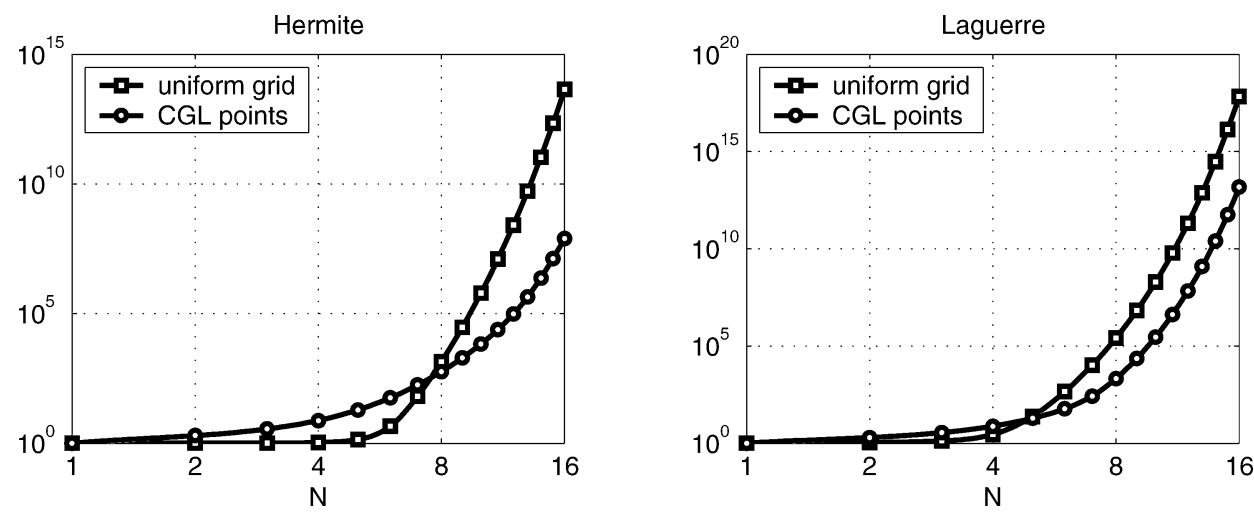

Fig. 2. Condition numbers of the matrices $Q_{H}$ and $Q_{L}$ versus $N$ for uniform grid and Chebyshev-Gauss-Lobatto (CGL) points.

We have plotted in Fig. 2 in double logarithmic scale the condition numbers of the matrices $Q_{H}$ and $Q_{L}$ versus $N$ for $1 \leqslant N \leqslant 16$ corresponding to the expansion (3.1) with the polynomials $l_{v}(x)$ based on uniform grid and Chebyshev-Gauss-Lobatto points in the interval $[-A, A]$. This figure corresponds to $n=2 N+1$ and to $r=8$ in (3.2) and (3.3). We can observe that these condition numbers grow rapidly with $N$ and as a result the approach based on the expansion (3.1) is only reliable for relatively small values of $N$. We can also observe that condition numbers based on uniform grid are larger than those based on Chebyshev-Gauss-Lobatto points for $N \geqslant 8$ in the Hermite case and for $N \geqslant 6$ in the Laguerre case. This is because the choice of Chebyshev points in the interval $[-A, A]$ limit the growth of the Lagrange polynomials as $N$ increases. For small $N$ the condition essentially depends on the clustering of the nodes $x_{i}$. We have also verified that the dependence of these condition numbers on the number of points $r$ of the Hermite or Laguerre quadrature rules is very weak.

\section{Numerical experiments}

In this section we present the results of numerical experiments using the approach developed in Sections 2 and 3. We have tested these methods on the problem (1.1) with the parameters $\sigma=0.5, \mu=0.2$, the function $f(\theta, t)$ given by

$$
f(\theta, t)=1+\cos \theta+F(t),
$$

where

$$
F(t)= \begin{cases}1, & 0 \leqslant t \leqslant 5 \\ 0, & t>5\end{cases}
$$

and the initial function $g(x)=0.1 x$. In Figs. 3 and 4 we present the results of numerical simulations using (2.9) and (2.10) with $A=50, T=10, N=100, M=100$, and $n=8$. We can observe the propagation of initial wave which then tends to the steady-state for large $t$ after the forcing term $F(t)$ assumes the value zero. In Fig. 5 we present the results of numerical experiments when we use piecewise constant instead of piecewise linear interpolation in the Euler-Hermite method (2.7). As discussed in Section 2 this introduces a discontinuity at $x=0$ which propagates with time $t$. This has a negative effect on 


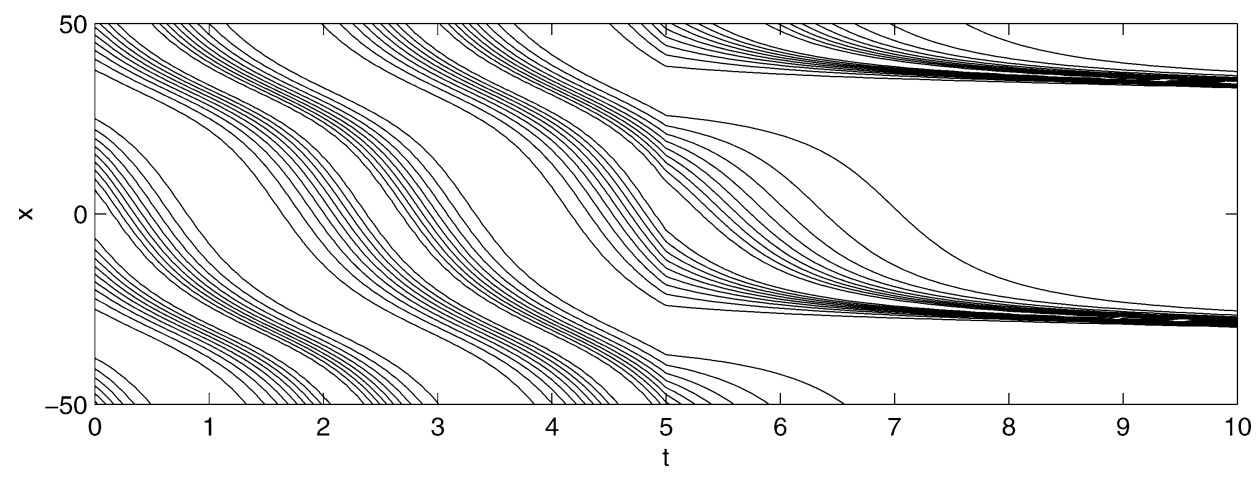

Fig. 3. Contour plots of $\cos \theta(x, t)$ for the Euler-Hermite method (2.9) with piecewise linear interpolation.

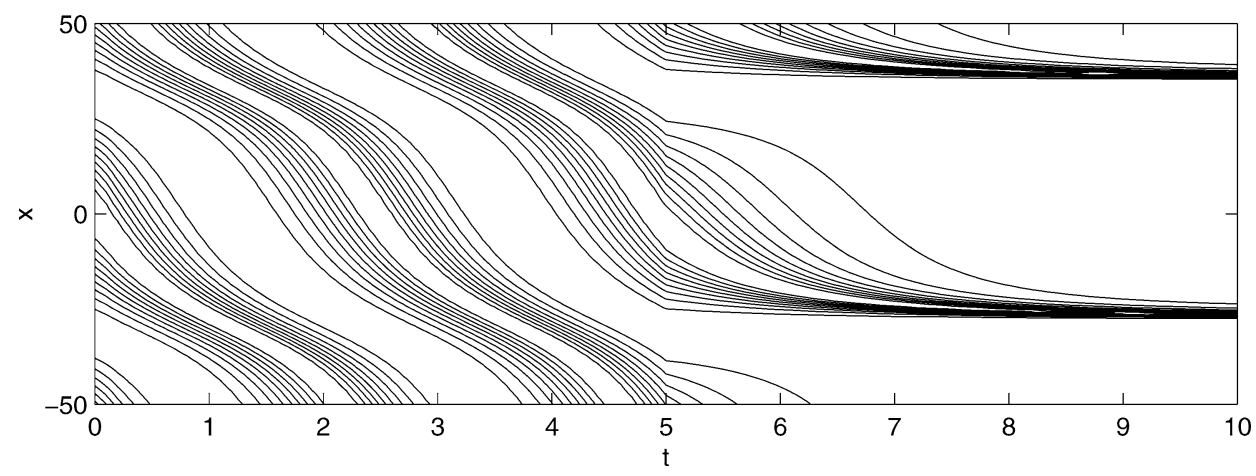

Fig. 4. Contour plots of $\cos \theta(x, t)$ for the Euler-Laguerre method (2.10) with piecewise linear interpolation.

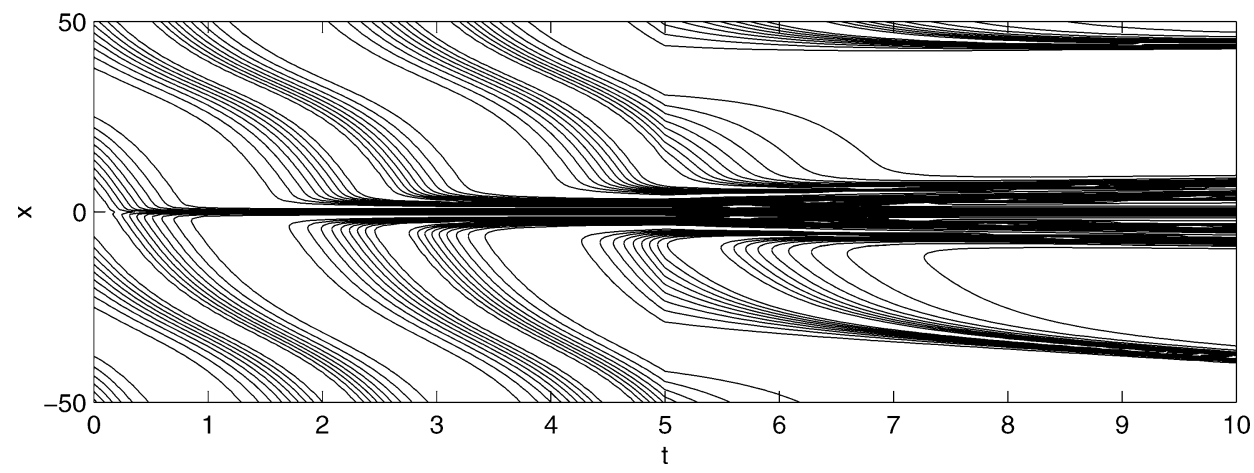

Fig. 5. Contour plots of $\cos \theta(x, t)$ for the Euler-Hermite method (2.7) with piecewise constant interpolation.

the accuracy of the resulting numerical scheme. In contrast, piecewise constant interpolation results in acceptable accuracy for the Euler-Laguerre method (2.8).

The results of numerical simulations using spectral collocation approach described in Section 3 are presented in Figs. 6 and 7. These figures correspond to $A=50, T=10, N=100, M=100, n=20$ and $r=8$. Since $2 N+1>n+1$ this results in the overdetermined systems of linear equations (3.6) which were then solved by least squares minimization. 


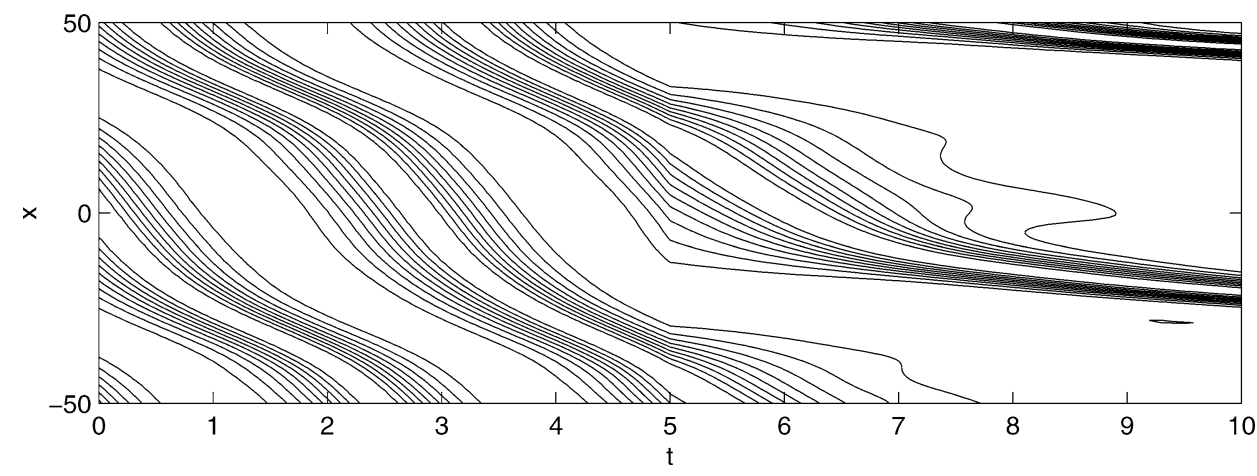

Fig. 6. Contour plots of $\cos \theta(x, t)$ for the spectral collocation method with $Q=Q_{H}$.

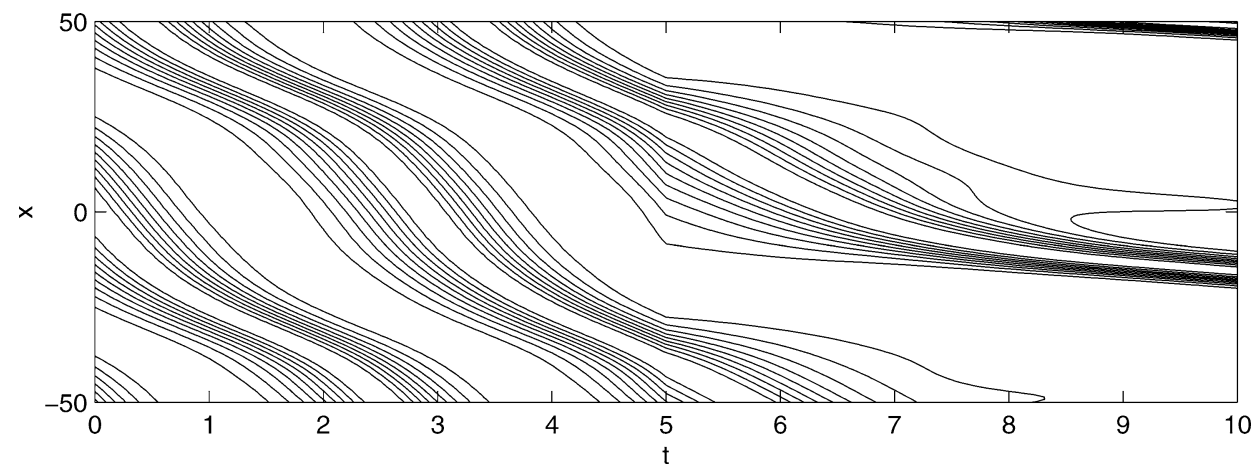

Fig. 7. Contour plots of $\cos \theta(x, t)$ for the spectral collocation method with $Q=Q_{L}$.

\section{Concluding remarks}

We proposed two new methods for the numerical solution of a Fredholm integro-differential equations modelling neural networks. The first approach is based on approximating the integral term by GaussHermite or Gauss Laguerre quadrature formula with linear interpolation to compute the approximation to the solution between the grid points in time. The second approach is based on representing the solution as the linear combination of Lagrange's fundamental polynomials with coefficients depending on time. In both approaches we use Euler method for time integration of the resulting implicit systems of ordinary differential equations.

Future work will address the numerical solution of Fredholm Volterra integro-differential equations modelling neural networks which also include the effects of memory which may be subject to noise occurring on different time scale than the system response. The model considered in this paper is a limiting case of such a system where the memory effects are suppressed.

\section{Acknowledgement}

The author wish to express his gratitude to anonymous referees for their helpful comments. 


\section{References}

[1] P.J. Davis, P. Rabinowitz, Methods of Numerical Integration, second ed., Academic Press, New York, 1984.

[2] M.T. Giraudo, L. Sacerdote, First entrance time distribution multimodality in a model neuron, Manuscript.

[3] S.A. Gourley, Y. Kuang, A delay reaction-diffusion model of the spread of bacteriophage infection, SIAM J. Appl. Math. 65 (2005) 550-565.

[4] F.C. Hoppensteadt, An Introduction to the Mathematics of Neurons. Modelling in the Frequency Domain, Cambridge University Press, New York, 1977.

[5] F.C. Hoppensteadt, $\dot{\theta}$-Networks, unpublished manuscript, Arizona State University.

[6] L.M. Riccardi, A. Di Crescenzo, V. Giorno, A.G. Nobile, An outline of theoretical and algorithmic approaches to first passage time problems with applications to biological modelling, Math. Japon. 50 (1999) 247-322. 